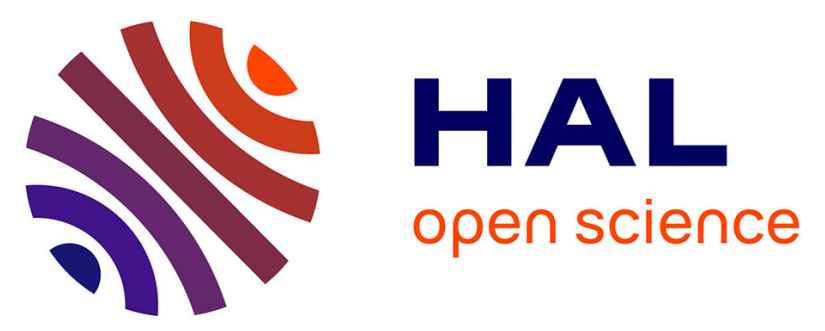

\title{
Influence à moyen terme (10-100 ans) d'un estran sableux macrotidal sur la stabilité d'un cordon de galets: la flèche de Cayeux (Picardie, France)/Medium term (10-100 yrs) influence of a macrotidal sandy foreshore on the stability of a gravel barrier: Cayeux spit (Picardy, France)
}

Franck Dolique, Edward J. Anthony

\section{- To cite this version:}

Franck Dolique, Edward J. Anthony. Influence à moyen terme (10-100 ans) d'un estran sableux macrotidal sur la stabilité d'un cordon de galets : la flèche de Cayeux (Picardie, France)/Medium term (10-100 yrs) influence of a macrotidal sandy foreshore on the stability of a gravel barrier: Cayeux spit (Picardy, France). Géomorphologie: relief, processus, environnement, 1999, 5 (1), pp.23-38. 10.3406/morfo.1999.973 . hal-02542768

\author{
HAL Id: hal-02542768 \\ https://hal.science/hal-02542768
}

Submitted on 8 Feb 2021

HAL is a multi-disciplinary open access archive for the deposit and dissemination of scientific research documents, whether they are published or not. The documents may come from teaching and research institutions in France or abroad, or from public or private research centers.
L'archive ouverte pluridisciplinaire HAL, est destinée au dépôt et à la diffusion de documents scientifiques de niveau recherche, publiés ou non, émanant des établissements d'enseignement et de recherche français ou étrangers, des laboratoires publics ou privés. 


\section{per \\ sq्e}

Géomorphologie : relief, processus, environnement

\section{Influence à moyen terme (10-100 ans) d'un estran sableux} macrotidal sur la stabilité d'un cordon de galets : la flèche de Cayeux (Picardie, France)/Medium term (10-100 yrs) influence of a macrotidal sandy foreshore on the stability of a gravel barrier: Cayeux spit (Picardy, France)

Monsieur Franck Dolique, Edward J. Anthony

\section{Citer ce document / Cite this document :}

Dolique Franck, Anthony Edward J. Influence à moyen terme (10-100 ans) d'un estran sableux macrotidal sur la stabilité d'un cordon de galets : la flèche de Cayeux (Picardie, France)/Medium term (10-100 yrs) influence of a macrotidal sandy foreshore on the stability of a gravel barrier: Cayeux spit (Picardy, France). In: Géomorphologie : relief, processus, environnement, Avril 1999, vol. 5, n¹. pp. 23-38;

doi : https://doi.org/10.3406/morfo.1999.973

https://www.persee.fr/doc/morfo_1266-5304_1999_num_5_1_973

Fichier pdf généré le 30/04/2018 


\title{
Résumé
}

Résumé L'évolution récente de la flèche de galets de Cayeux, en Picardie, France, est une illustration des ajustements morphologiques rapides (<100 ans) que peuvent connaître ces types d'accumulations constituées de dépôts grossiers lorsque leur bilan sédimentaire est perturbé par des actions humaines. La flèche est associée à un important estran sableux plat, plus ou moins large, affecté par de forts courants de marée (marnage moyen de vive-eau = $10 \mathrm{~m}$ ) dominés par un courant résiduel du flot dirigé vers l'estuaire de la Somme. Malgré de nombreuses études sur la dynamique de la flèche de galets depuis plus d'un siècle, l'influence potentielle de ce bas estran sableux sur cette flèche n'a pas suscité beaucoup de curiosité. Étroit $(<1 \mathrm{~km})$ dans le secteur amont-dérive où la flèche a été fortement érodée, l'estran sableux s'élargit progressivement pour atteindre plus de $5 \mathrm{~km}$ à l'entrée de l'estuaire de la Somme. Cette évolution longitudinale témoigne d'une importante accretion en zone distale largement conditionnée par des apports de sables du large. En revanche, la flèche de galets montre, dans cette zone d'accrétion distale de l'estran sableux, des secteurs en érosion, en accretion ou stables. Cette alternance est conditionnée par la houle et par des gradients de dérive littorale qui déterminent la répartition longitudinale des galets originaires de l'érosion de falaises côtières normano-picardes au sud. Elle montre que l'estran sableux n'est pas fondamentalement responsable des variations dynamiques longitudinales de la flèche. Toutefois, il modère ou favorise l'érosion, le transport et le dépôt des galets, et constitue de ce fait un élément important dans l'explication de certaines tendances morphodynamiques récentes esquissées par la flèche.

\begin{abstract}
Abstract The gravel spit of Bas -Champs, Picardy, France, is fronted by aflat macrotidal sandy foreshore that considerably widens near the Somme estuary. The recent evolution of this spit highlights the rapid morphological adjustments of such deposits to medium term (<102 yrs) sediment budget perturbations, while illustrating their response to changes in sand supply affecting the foreshore. These perturbations have induced division of the former single gravel cell characterizing this spit into one major and one minor cell. These processes are endangering the integrity of the spit, which has broken down into an unstable feature exhibiting longshore alternations of erosion and accumulation. The sandy foreshore also shows updrift erosion and important downdrift aggradation in the vicinity of the Somme estuary. By modulating the duration of wave action on the steep gravel beach face during the tidal cycle, the sandy foreshore moderates or reinforces the gravel transport gradients but has not played a fundamental role in the division of the gravel spit sediment cell. Cell division has occurred solely in response to longshore gradients in gravel availability.
\end{abstract}

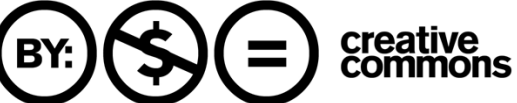




\title{
Influence à moyen terme (10-100 ans) d'un estran sableux macrotidal sur la stabilité d'un cordon de galets : la flèche de Cayeux (Picardie, France)
}

\author{
Medium term (10-10z yrs) influence of a macrotidal sandy foreshore on the sta- \\ bility of a gravel barrier: Cayeux spit (Picardy, France)
}

Franck DOLIQUE* et Edward J. ANTHONY*

\begin{abstract}
Résumé
L'évolution récente de la flèche de galets de Cayeux, en Picardie. France, est une illustration des ajustements morphologiques rapides $(<100)$ ans) que peuvent connaître ces types d'accumulations constituées de dépôts grossiers lorsque leur bilan sédimentaire est perturbé par des actions humaines. La flèche est associée à un important estran sableux plat, plus ou moins large, affecté par de forts courants de marée ( marnage moyen de vive-eau $=10 \mathrm{~m}$ ) dominés par un courant résiduel du flot dirigé vers l'estuaire de la Somme. Malgré de nombreuses études sur la dynamique de la flèche de galets depuis plus d'un siècle, l'influence potenticlle de ce bas estran sableux sur cette flèche n`a pas suscité beaucoup de curiosité. Étroit $(<1 \mathrm{~km})$ dans le secteur amont-dérive où la flèche a été fortement érodéc. l'estran sableux s'élargit progressivement pour atteindre plus de $5 \mathrm{~km}$ à l'entrée de l'estuaire de la Somme. Cette évolution longitudinale témoigne d'une importante accrétion en zone distale largement conditionnée par des apports de sables du large. En revanche, la flèche de galets montre, dans cette zone d'accrétion distale de l'estran sableux, des secteurs en érosion, en accrétion ou stables. Cette alternance est conditionnée par la houle et par des gradients de dérive littorale qui déterminent la répartition longitudinale des galets originaires de l'érosion de falaises côtières normano-picardes au sud. Elle montre que l'estran sableux n'est pas fondamentalement responsable des variations dynamiques longitudinales de la flèche. Toutefois, il modère ou favorise l'érosion, le transport et le dépôt des galets. et constitue de ce fait un élément important dans l'explication de certaines tendances morphodynamiques récentes esquissées par la flèche.
\end{abstract}

Mots clés : flèche de galets, estran sableux. dynamique sédimentaire, Picardie, France.

\section{Abstract}

The gravel spit of Bas-Champs, Picardy, France, is fromted by a flat macrotidal sandy foreshore that considerably widens near the Somme estuary: The recent evolution of this spit highlights the rapid morphological adjustments of such deposits to medium term $\left(<10^{2}\right.$ yrs $)$ sediment budget perturbations, while illustrating their response to changes in sand supply affecting the foreshore. These perturbations have induced division of the former single gravel cell characterising this spit into one major and one minor cell. These processes are endangering the integrity of the spit, which has broken down into an unstable feature exhibiting longshore alternations of erosion and accumulation. The sandy foreshore also shows updrift erosion and important donndrift aggradation in the vicinity of the Somme estuary: By modulating the duration of wave action on the steep gravel beach face during the tidal cacle, the sandy foreshore moderates or reinforces the gravel transport gradients but has not played a fundamental role in the division of the gratel spit sediment cell. Cell division has occurred solely in response to longshore gradients in gravel arailability.

Key words: gravel barrier spit. sandy foreshore. sediment dynamics, Picardy. France.

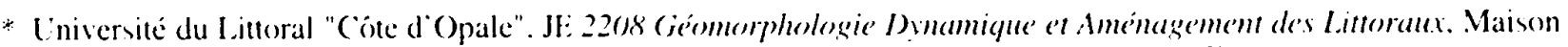
de la Recherche en Environnement Naturel. 28. Avenue Foch. B.P. 59, F-62930) Wimereux. France.
} 


\section{Abridged English version}

Gravel barriers are prominent coastal deposits that may play an essential protective role against storms. Recent studies on these deposits have focussed on the short (1-10 yrs) to medium $\left(10-10^{2}\right.$ yrs) term morphosedimentary adjustments they exhibit under conditions of diminished sediment supply or sea-level change leg., Forbes et al., 1995; Orford and Carter, 1995; Anthony et al., 1998). One of the best known examples of a gravel barrier in France is the Bas-Champs spit in Picardy. The spit has been sourced by erosion of the Picardy and Upper Normandy coasts further south, where episodic failures of chalk cliffs liberated flint clasts that were transported northwards by longshore drift generated by short waves from a dominantly westerly direction. This supply is now almost totally cut off by harbour breakwaters and cliff protection works. As a result, portions of the spit have been undergoing severe erosion, thus posing a serious socio-economic threat to part of the Picardy coast. The spit rests on, and is fronted by, a flat macrotidal (mean spring tide range $=10 \mathrm{~m}$ ) sandy foreshore that widens from less than $1 \mathrm{~km}$ in the proximal segment of the spit to over $5 \mathrm{~km}$ in the distal segment in the north near the Somme estuary; a major sand trap on this coast. The paper discusses the processes of recent spit evolution in terms of medium term $\left(<10^{2}\right.$ yrs) sediment budget changes, and focusses on the influence of changes in elevation of the sandy foreshore on the spit. The work is based on extensive data collection from field n'ork involving topographic, sedimentological and hydrodynamic surveys, and from old maps, bathymetric charts, written and pictorial documents, land-use surveys, and aerial photographs.

The spit has broken down into an unstable feature exhibiting longshore alternations of erosion and accumulation, as the former single sediment cell characterizing gravel transport gradients has broken down into two cells. Changes in the morphology of the sandy foreshore have also occurred as a result of anthropogenic perturbation of longshore sand supply in the south and especially of large-scale accumulation of sand near the Somme estuary. The northward transport of sand towards the Somme estuary is due mainly to strong flood-dominated tidal currents. While a considerable amount of research has been devoted to the longshore changes that have dramatically affected the spit over the last century, very little attention has been given to the potential influence of longshore changes in the width and elevation of the sandy foreshore on the dynamics of the spit. Globally, we show that the role of sandy foreshore elevation and width changes is subordinate to that of the gravel sediment supply itself and the way its accumulation alongshore has modulated the wave field, resulting in a bi-cellular gravel barrier structure. Gravel is transported alongshore by wave action along the steep beach face. Gravel entrainment is therefore particularly sensitive to longshore wave transport gradients resulting from differences in refraction and dissipation. These have however been affected by evolution of the sandy foreshore. Net sand influx towards the north enhances wave energy dissipation and therefore less active transport of gravel, thus favouring spit progradation. While more dissipative conditions have globally characterized this northern, downdrift zone, cell segmentation has nevertheless occurred, because the prograding spit in this sector sequesters all the gravel, depriving the downdrift zone. Erosion of this deprived zone has however been strongly constrained over the past years by massive foreshore sand accumulation. Such sand accumulation has fed dunes that overwhelm the gravel barrier in this sector Observations over the last decade show that the short distal part of the spit characterized by the second sediment cell is now in a state of relative stability by virtue of the protection against storm waves offered by the highly dissipative sandy foreshore. The role of wide sandy foreshores associated with gravel barriers in macrotidal settings should be an important consideration in explaining some of the dynamic longshore trends exhibited by these barriers. 


\section{INTRODUCTION}

Les cordons de galets sont des formes d'accumulation côtières dont le rôle de protection contre les tempêtes est souvent essentiel sur beaucoup de littoraux. Sur des côtes méso- à macrotidales (marnage moyen de vive-eau $>3 \mathrm{~m}$ ), ces corps sédimentaires sont souvent associés à un estran sableux plus ou moins large, en fonction de l'abondance du sable et du marnage. L'évolution morphologique de ce bas estran sableux peut, à terme, avoir des répercussions importantes sur la stabilité du cordon de galets, comme l'ont suggéré Carter et Orford (1993), en modulant les conditions hydrodynamiques et de dépôt. Les relations entre ces deux corps sédimentaires sont toutefois assez peu connues (Forbes et al., 1995). En effet, les nombreuses études effectuées ces dernières années sur les littoraux à galets ont mis l'accent sur la manière dont les cordons s'ajustent morphologiquement à court terme ( 1 à 10 ans) et à moyen terme (10 à 100 ans) à des fluctuations d'apports de galets ou des forçages météorologiques et eustatiques (Forbes et al., 1995 ; Orford et Carter, 1995 ; Orford et al., 1996 ; Anthony et Cohen, 1996 ; Anthony et al., 1998).

Un bon exemple de système mixte cordon de galets-estran sableux est celui de la flèche des Bas-Champs, en Picardie (fig. 1). Cette flèche, une des plus célèbres mais aussi une des plus importantes, en France, forme un haut estran de galets associé à un large estran sableux qui constitue un bas de plage intertidal très plat, aux abords de la baie de Somme (Dolique, 1998a). La flèche ainsi que les Bas-Champs en arrière qu'elle protège ont une fonction socio-économique importante sur la côte picarde et sont le théâtre de sévères conflits d'intérêts (Dolique, 1998b). D'importants travaux de génie côtier et des extractions de granulats sur les côtes normandes et picardes depuis la première moitié du $\mathrm{XX}^{\mathrm{e}}$ siècle ont perturbé les transits de galets et l'alimentation de la section distale de la flèche. Le problème d'érosion qui en a découlé a fait l'objet de nombreux rapports et études, dont le plus récent est celui de la SOGREAH (1995a). Il complète une série longue de travaux (Briquet, 1930 ; Dallery, 1955 ; Regrain, 1970, 1971, 1992 ; Migniot et Bellesort, 1974 ; Hascoët, 1988 ; Beauchamp, 1994) qui ont bien mis en évidence la dynamique de l'érosion de ce cordon depuis la fin du siècle dernier. Regrain et al. (1979) ont montré en particulier qu'une migration progressive vers le nord du secteur d'érosion s'était produite suite à la mise en place des ouvrages d'ingénierie plus au sud. Par contre, les relations dynamiques et morphosédimentaires entre le bas estran sableux et le haut estran de galets n'ont pas jusqu'ici fait l'objet d'études détaillées. Du fait de son rôle important de protection des terrains agricoles des Bas-Champs et des zones urbanisées contre la mer, l'attention portée sur le cordon lui-même est compréhensible, même si elle semble démesurée, mais il est important d'essayer de cerner l'influence que la masse imposante de sables, qui s'est accumulée au voisinage de l'estuaire de la Somme, a pu exercer sur la dynamique de ce cordon. Nous rappellerons brièvement l'évolution récente (depuis un siècle) du cordon avant d'aborder la manière dont l'estran sableux a pu influencer cette évolution.

\section{CADRE ENVIRONNEMENTAL}

La flèche de Cayeux s'est développée en zone terminale d’une cellule sédimentaire entre le Cap d'Antifer en Haute-Normandie et l'estuaire de la Somme en Picardie 


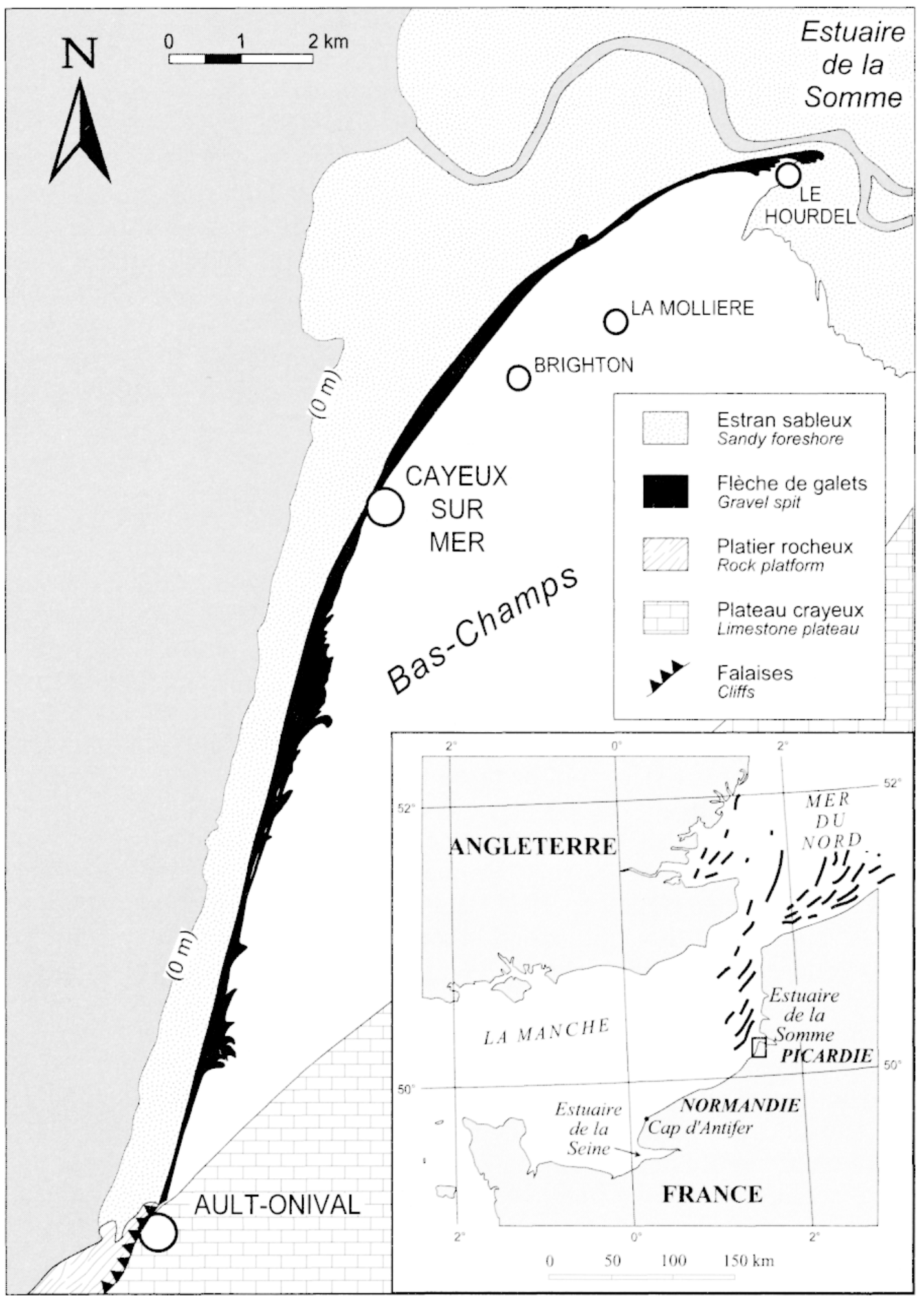

Fig. 1 - La flèche de Cayeux en Picardie. L'encart montre le contexte régional et la localisation des bancs de sables près des côtes françaises.

Fig. I - Cayeux spit, Picardy, France. Inset shows regional setting and sand banks adjacent to the French coast. 
(fig. 1). La flèche est ancrée à son extrémité proximale à une falaise crayeuse (fig. 1) et repose sur des dépôts sableux qui constituent la continuité de l'avant-côte sableuse de la Manche. L'âge de ce cordon n'est pas connu avec certitude. Dupont (1994) a réalisé une datation ${ }^{14} \mathrm{C}$ de $2440 \pm 150$ ans B.P. sur des coquilles de Cerastoderma edule, une faune de milieu estuarien, en bordure de l'actuelle falaise morte derrière les BasChamps. Il est vraisemblable, au vu de cet âge tardif, qui doit être confirmé toutefois par des datations plus nombreuses, que le développement optimal de la flèche ait été conditionné par l'accrétion d'un estran sableux jusqu'à un niveau suffisamment élevé pour constituer une plate-forme sur laquelle ce corps de galets s'est étendu. Le cordon a été pendant longtemps alimenté en galets par l'érosion des falaises de craie, riches en rognons et lits siliceux. Les galets issus du tri et du transport par roulement de ces dépôts siliceux étaient acheminés vers le nord-est par les houles dominantes de secteur ouest (Costa, 1997 ; Dolique, 1998a). Le cordon évolue dans un contexte macrotidal, avec un marnage compris entre $10 \mathrm{~m}$ et $10,5 \mathrm{~m}$ en vive-eau. Cet important marnage est associé à des courants de marée puissants qui assurent l'essentiel du transport des sables au large et près de la côte (Grochowski et al., 1993a, 1993b).

\section{MÉTHODES}

L'aménagement, déjà ancien, des Bas-Champs picards a suscité un nombre important de documents écrits et cartographiques qui ont servi à dégager les tendances évolutives morphosédimentaires de la flèche de galets et du bas de plage sableux au cours du $\mathrm{xx}^{\mathrm{e}}$ siècle. Ces données, couplées à onze séries successives de photographies aériennes IGN s'échelonnant de 1939 à 1997, ont été largement exploitées par les différents auteurs et acteurs ayant travaillé sur ce littoral. Pour cerner plus spécifiquement l'évolution de l'estran sableux depuis un siècle, nous avons consulté des cartes postales anciennes (Dolique, 1998c) et des cartes bathymétriques remontant au début du $\mathrm{xx}^{\mathrm{e}}$ siècle. À plus courte échelle temporelle, notre travail de terrain, effectué depuis 1991, a porté sur plusieurs levés topographiques, des analyses granulométriques, diverses observations sur les formes, les figures sédimentaires et la dynamique, ainsi que sur des mesures de courants et de houles acquises avec un courantomètre électromagnétique S4 à capteur de pression. Les profils topographiques présentés dans ce travail ont été effectués en 1996 et 1997 à l'aide d'une Station Electronique Totale Leica TC60), du haut estran de galets au bas estran sableux sur une distance variant entre $100 \mathrm{~m}$ et $130 \mathrm{~m}$. Les levés ont été reliés à des points de calage IGN 69 du réseau géodésique national. Une analyse granulométrique portant sur une trentaine d'échantillons le long de trois transects de $200 \mathrm{~m}$ sur le bas de plage au droit des parties proximale, centrale et distale de la flèche a servi à cerner les caractéristiques des sables.

\section{MORPHOLOGIE ET BILAN SÉdIMENTAIRE DE LA FLĖCHE : UN BREF RAPPEL}

La synthèse des divers travaux sur la flèche de Cayeux, complétée par la consultation des photographies aériennes récentes (séries IGN 1991, 1995 et 1997) permet d'identifier cinq secteurs morphologiques longitudinaux sur la base de la stabilité dynamique de la flèche de galets (Dolique et Anthony, 1998). Ces secteurs sont indiqués sur la fig. 2. Une réduction drastique des apports de galets, du fait de l'implanta- 


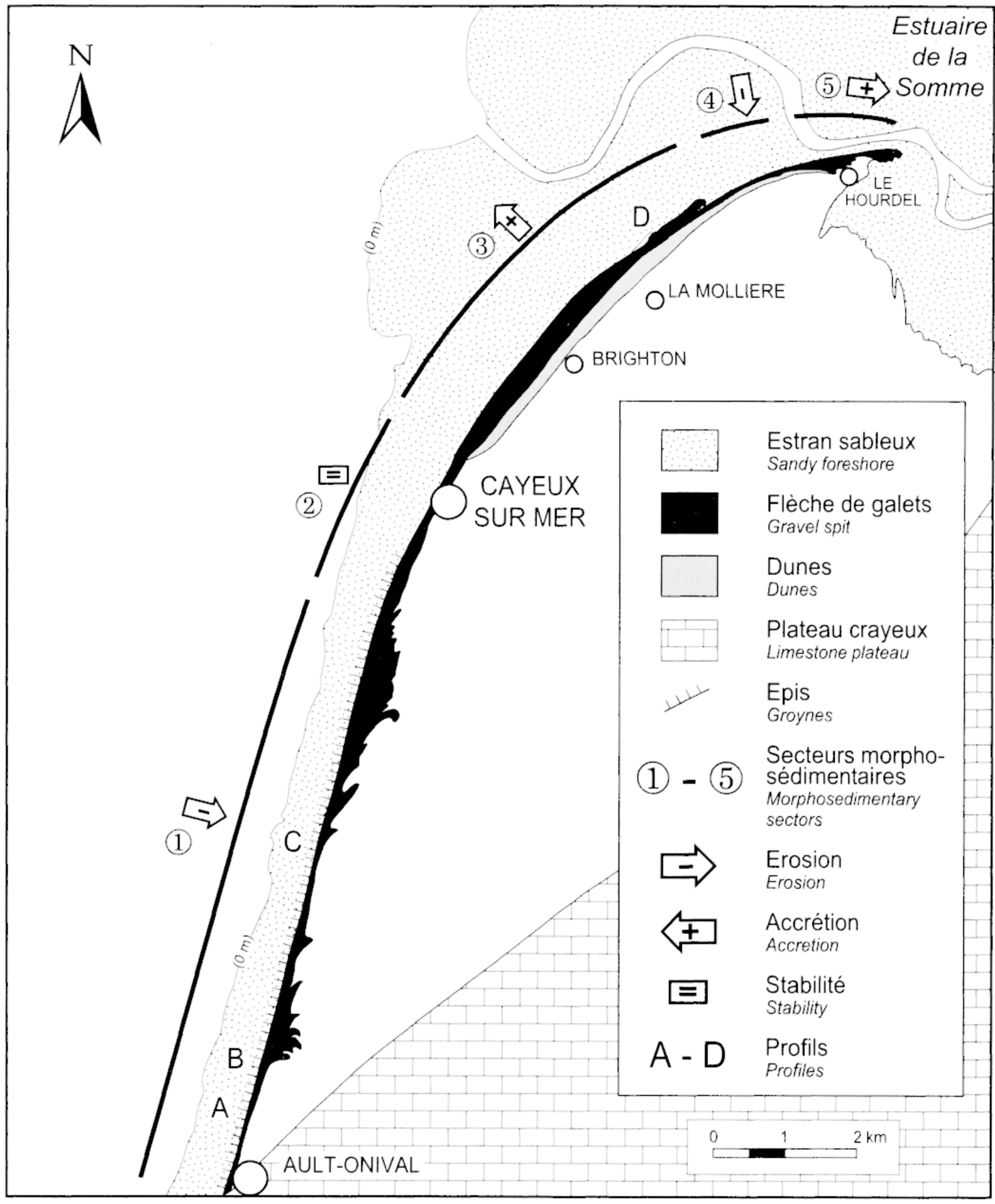

Fig. 2 - Secteurs morphologiques longitudinaux de la flèche de Cayeux. Ces secteurs ont été identifiés en fonction de l'état dynamique du rivage de galets. Noter l'élargissement considérable de l'estran sableux vers le nord.

Fig. 2 - Morphological sectors of Cayeux spit. These sectors have been identified from the dynamic longshore status of the gravel shoreline. Note the considerable widening of the sandy foreshore tow'ards the north. 
tion d'ouvrages d'ingénierie en amont de la flèche, notamment les jetées portuaires de Dieppe et du Tréport et les infrastructures de la centrale nucléaire de Penly, a entraîné l'érosion du secteur 1. Cette érosion est combattue aujourd'hui par l'implantation de nombreux épis (photo 1) et par des rechargements en galets. Les observations effectuées ces dernières années montrent que pratiquement toute la charge de galets transportée le long de la plage transite par le secteur 2 pour se déposer dans le secteur 3 (fig. 2) qui fonctionne ainsi comme un puits sédimentaire efficace s'engraissant par le biais de crochets de cordons successifs dont la formation se poursuit actuellement (photo 2). Cet engraissement local entraîne un déficit total en galets dans le secteur 4 qui, tout comme le secteur 5, jadis la zone d'accumulation distale de la flèche, ne reçoivent plus guère de galets aujourd'hui. Ces secteurs, qui étaient respectivement en érosion et en accrétion il y a encore quelques années, montrent aujourd'hui une certaine stabilité dont les raisons sont à rechercher dans une protection offerte par l'estran sableux.

À partir d'un travail initial de Beauchesne et Courtois (1967), Queffeullou (1992) a calculé des taux de transit potentiel annuel de galets par la dérive littorale le long de cette partie de la côte picarde. Sur la base de ces taux potentiels et des taux de transit réels que nous avons dégagés à partir du suivi des cinq secteurs morphologiques montrés dans la fig. 2, nous avons identifié deux cellules de transit de galets (fig. 3). Ces cellules définissent des gradients longitudinaux d'érosion, de transport et de sédimentation à moyen et à long terme (de 10 à $10^{3}$ ans) liés aux agents hydrodynamiques (May et Tanner, 1973). Elles permettent de bien cerner les différences longitudinales du bilan sédimentaire littoral à ces échelles temporelles, et constituent donc un outil de première importance pour l'aménagement du littoral (Bray et al., 1995).

La première cellule recouvre les secteurs morphologiques 1 à 3 et la seconde, plus courte, les secteurs 4 et 5 dans la fig. 2. Les divers documents cartographiques et les écrits montrent que, jusqu'au début du $\mathrm{XX}^{\mathrm{e}}$ siècle, la flèche des Bas-Champs était caractérisée par une cellule sédimentaire unique comportant deux secteurs : un long couloir stable, de transit de galets recouvrant les secteurs actuels 1 à 2 , et un court secteur d'accumulation en fin de cellule, correspondant aux secteurs 3 à 5 actuels où se sont formées de nombreuses rides d'accumulation de galets (Briquet, 1930). Coupée des stocks de galets frais provenant de la zone d'alimentation amont-dérive de cette cellule, c'est-à-dire des falaises normano-picardes, la flèche a subi une réorganisation interne majeure de son propre stock de galets. En effet, pour assurer la continuité du transport sédimentaire, le courant de dérive littorale a provoqué l'érosion de la partie proximale de la flèche (secteur 1, photo 1) pour alimenter le reste. L'essentiel de ces galets remaniés a été piégé dans le secteur 3 où se sont constituées plusieurs rides de galets en crochets depuis les années 1920 (Briquet, 1930). L'accumulation de tels crochets successifs est un processus qui s'auto-entretient puisqu'elle perturbe le champ de houle, entraînant une réfraction accrue, une baisse d'énergie des vagues par mètre linéaire de plage, un ralentissement de la dérive et le dépôt des galets. Le même dépôt privilégié dans ce secteur prive la partie aval-dérive de galets, entraînant la division de l'ancienne cellule unique en deux cellules actuelles. Une telle structure bi-cellulaire diffère radicalement des structures unicellulaires classiques que l'on rencontre sur des flèches à pointe libre. que ce soient des flèches sableuses ou constituées de galets. 


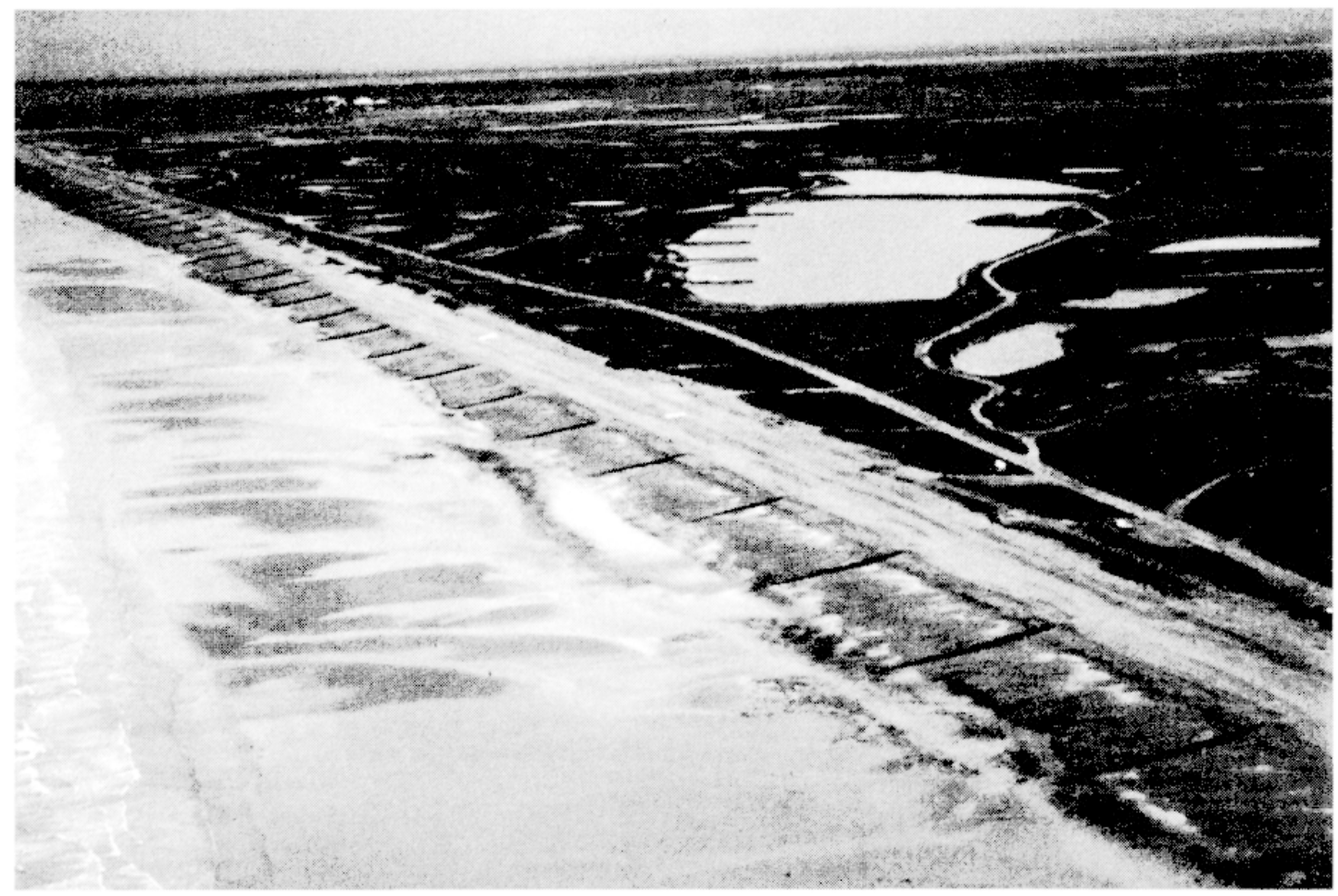

Photo 1 - Secteur proximal (secteur 1, fig. 2) de la flèche en érosion. Ce secteur est défendu aujourd'hui par une batterie d'épis et par des rechargements en galets.

Photo I - Proximal eroding sector (sector 1, fig. 2) of the spit. This sector is presently protected by a grosne field and frequently renourished in gravel.

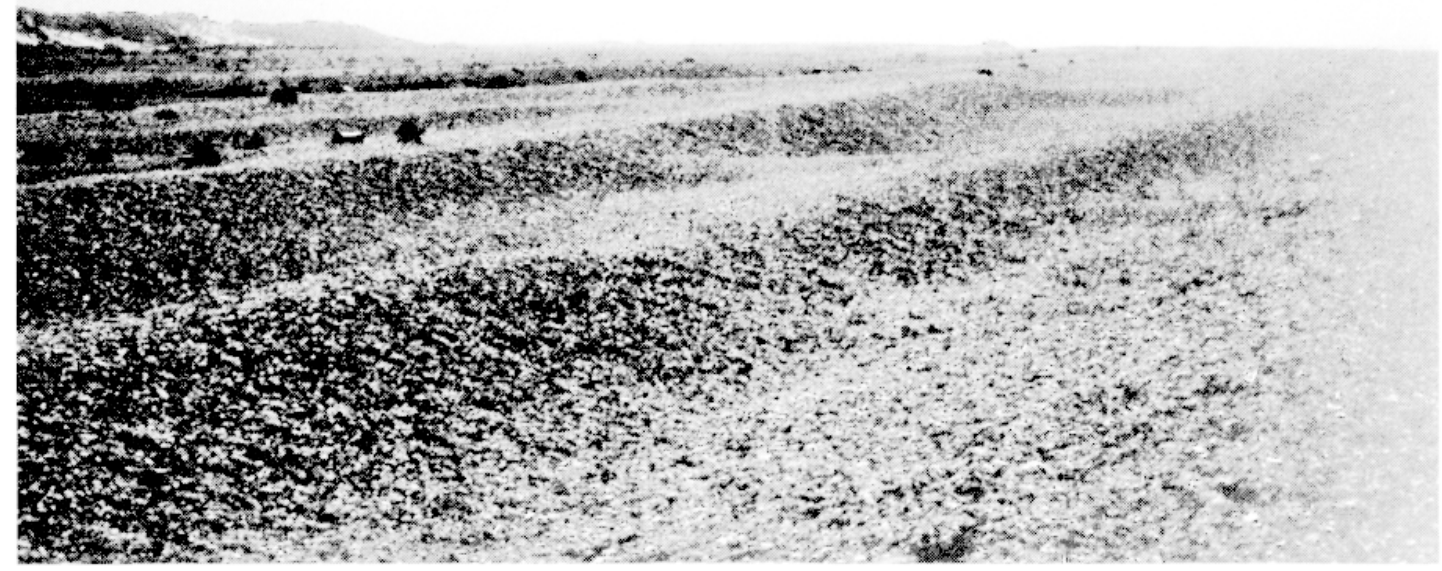

Photo 2 - Rides de galets successives témoignant de la progradation active de la flèche dans le secteur 3 (fig. 2).

Photo 2 - Successive gravel ridges illustrating active spit progradation in sector 3 (fig. 2). 


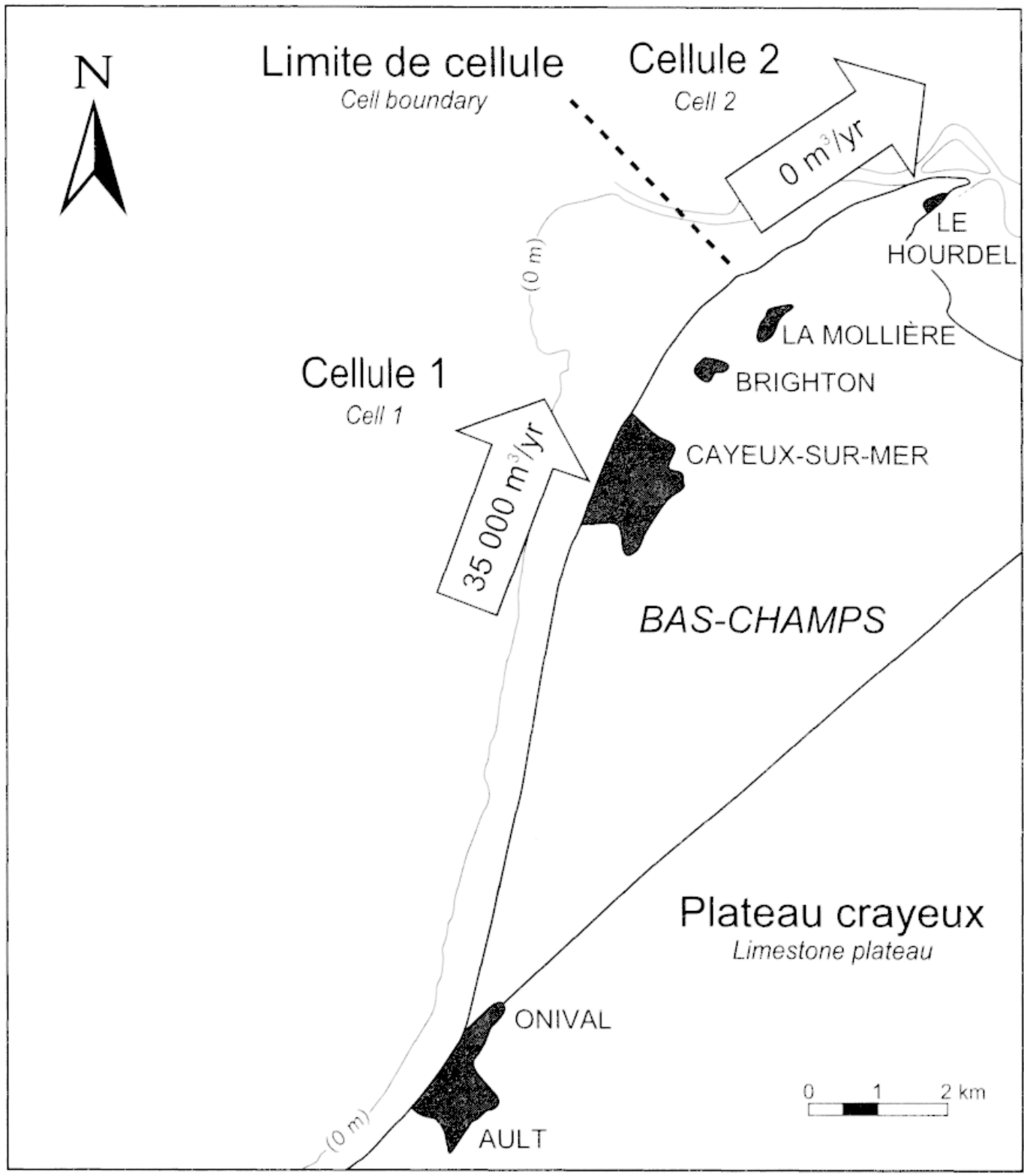

Fig. 3 - Transits estimés de galets et cellules sédimentaires actuelles associées à la flèche.

Fig. 3 - Estimated gravel drift rates and present sediment cells associated with the spit.

Les processus décrits ci-dessus expliquent aussi l'érosion. dans un passé récent, du secteur 4 (fig. 2) où la capacité de transport de la dérive augmentait de nouveau. L'érosion du secteur 4 était due au fait que la dérive se rechargeait de nouveau pour alimenter l'extension de la flèche vers l'intérieur de la Baie de Somme. Ces dernières années. tandis que l'érosion de la partie proximale de la flèche se poursuit, alimentant l'accrétion du secteur 3. les secteurs 4 et 5 sont dans un état de stabilité relative. 


\section{L'ESTRAN SABLEUX : MORPHOLOGIE ET ORIGINE DES SABLES}

Le bas estran sableux (fig. 2) se prolonge vers la partie méridionale de l'important delta de marée de l'estuaire de la Somme. Il est constitué de sables moyens à fins (médiane granulométrique d'environ $0,2 \mathrm{~mm}$ ). Sa largeur, définie par la courbe bathymétrique $0 \mathrm{~m}$ (à ne pas confondre avec les cotes IGN 69), passe de $1 \mathrm{~km}$ au sud, entre Ault-Onival et Cayeux-sur-Mer, à plus de $5 \mathrm{~km}$ au nord de Cayeux-sur-Mer (fig. 2), se confondant avec l'importante accumulation sableuse de l'estuaire de la Somme. Cette évolution va de pair avec l'élévation du contact entre le bas estran sableux et le haut estran de galets qui passe de -1 m IGN 69 à Onival à environ +2 m IGN 69 à la Mollière (fig. 4). Ces profils montrent par ailleurs la transition nette entre la plage de galets raide et l'estran sableux extrêmement plat. L'altitude de la crête de la flèche varie entre $5 \mathrm{~m}$ et $10 \mathrm{~m}$ IGN 69.

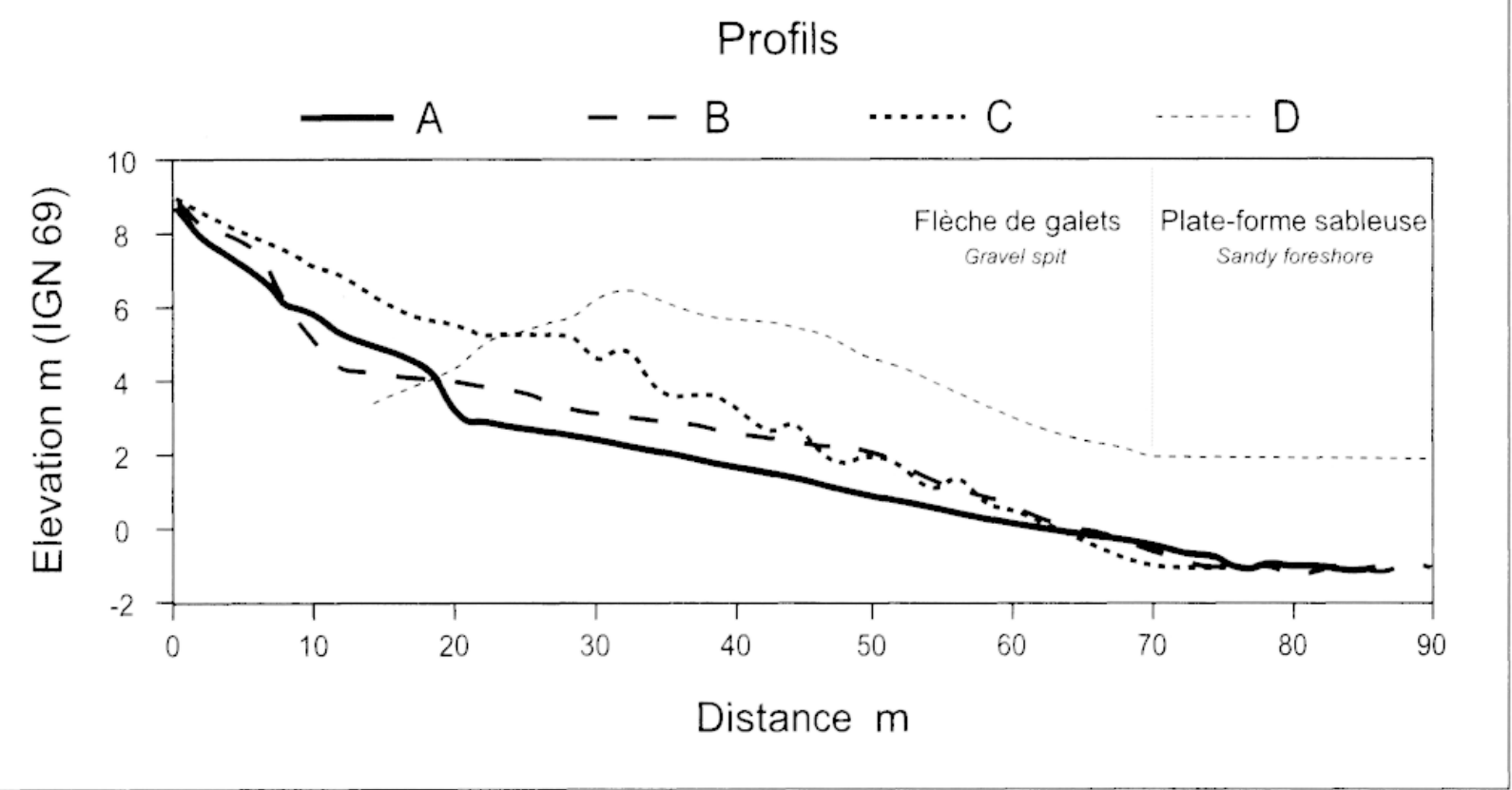

Fig. 4 - Profils transversaux A, B, C, D, localisés sur la fig. 2, montrant la transition abrupte entre le haut estran de galets à pente raide et le bas estran sableux. Noter la différence d'altitude de la surface de l'estran entre le profil A, au nord de Cayeux-sur-Mer et les trois autres profils au sud de cette localité.

Fig. 4 - Cross-shore profiles A, B, C, D, (see fig. 1 for locations) showing the abrupt change in slope between the steep gravel spit face and the flat sandy foreshore. Note the increase in foreshore elevation at profile A north of Cayeux-sur-Mer.

De récentes modélisations numériques sur les transports sédimentaires d'avant-côte dans cette zone de la Manche, effectuées dans le cadre du Programme MAST FluxManche (1990-1993), ont montré l'existence d'un flux de sables originaires de la Manche, circulant jusqu'en Mer du Nord, dans un couloir le long des côtes françaises 
(Grochowski et al., 1993a, 1993b). Le couloir commence précisément aux environs de l'estuaire de la Somme. Le transport des sables se fait sous l'effet de courants de marée dont le courant résiduel est dominé par le flot. Cette domination du flot est mise en évidence par des mesures courantométriques que nous avons effectuées sur le bas de plage au niveau de Cayeux-sur-Mer à plusieurs reprises en 1997 (Dolique, 1998a). Elle est aussi confirmée par un réseau dense de courantomètres déployés sur la plage de Merlimont, $30 \mathrm{~km}$ plus au nord dans le Pas-de-Calais (Levoy et al., 1998).

Les sables sembleraient emprunter le sommet parfois peu profond $(<10 \mathrm{~m})$ des nombreux bancs de sables au large des côtes du Nord de la France (fig. 1). Ces vastes corps sédimentaires sont considérés comme étant essentiellement des corps "allocycliques", c'est-à-dire des corps composites dus conjointement à des variations eustatiques et à des processus hydrodynamiques. Ils comprennent à la base des dépôts fluviaux ou estuariens surmontés de sables marins mis en place lors de la transgression flandrienne (De Batist et al., 1993 ; Tessier, 1997) et façonnés ensuite par des courants de marée soutenus et par des houles de tempêtes périodiques. Le couloir de transport qui longe cette côte sableuse a pu jouer un rôle primordial dans l'alimentation sableuse des massifs dunaires et des estuaires depuis la Somme jusqu'en Mer du Nord au cours de l'Holocène (Anthony, 1998).

L'évolution morphologique récente de l'estran sableux met en évidence un transfert longitudinal de sables assuré par des courants de marée et de houle combinés. Par ailleurs, cet estran a été vraisemblablement alimenté aussi par des apports directs de sables en provenance du large. Un rapport technique de la SOGREAH (1995a) montre un abaissement net, de l'ordre d'un mètre, du bas estran sableux dans la partie proximale de la flèche, entre Ault-Onival et Cayeux-sur-Mer, et en contre-partie, une nette agradation de l'estran au nord de Cayeux-sur-Mer. Cette évolution est confirmée par une comparaison des profils que nous avons levés en 1997 à ceux de la SOGREAH (1995a) levés dès 1965. Elle indique d'abord une érosion dans la partie proximale non compensée par des entrées de sables provenant du large ou des côtes picardes et normandes plus au sud. Elle laisse à penser aussi que les structures d'ingénierie côtière au sud d'Ault-Onival, notamment les jetées portuaires, perturbent le transit des sables dans la zone d'estran proche du rivage, et que les volumes de sables en provenance du sud sont peu importants. En effet, de longs secteurs de cette côte à falaises comportent un platier rocheux en guise d'estran. La comparaison de cartes bathymétriques du SHOM montre aussi que l'estran sableux s'est nettement élargi dans la partie distale de la flèche vers la Somme (photo 3). L'estuaire de la Somme est connu pour être un redoutable puits sédimentaire qui, d'après des études récentes de modélisation, piège jusqu'à $700.000 \mathrm{~m}^{3}$ de sables par an (SOGREAH, 1995b; Hachache, 1998). Cette accumulation massive en zone distale a vraisemblablement été alimentée par l'érosion du bas estran sableux de la partie proximale au sud et surtout par des apports directs de sables du large.

Remaniés vers la terre par le vent à marée basse, les sables accumulés dans la partie distale de la flèche des Bas-Champs de Picardie ont alimenté, depuis les années 1920, un important massif dunaire entre Cayeux-sur-Mer et Le Hourdel (fig. 2). Ces apports de sables ont été suffisamment importants dans les années 1920 et 1930 pour édifier, en 20 ans, au-devant du Casino de Brighton-les-Pins. une dune de $18 \mathrm{~m}$ de haut (Dolique, 1998c). 


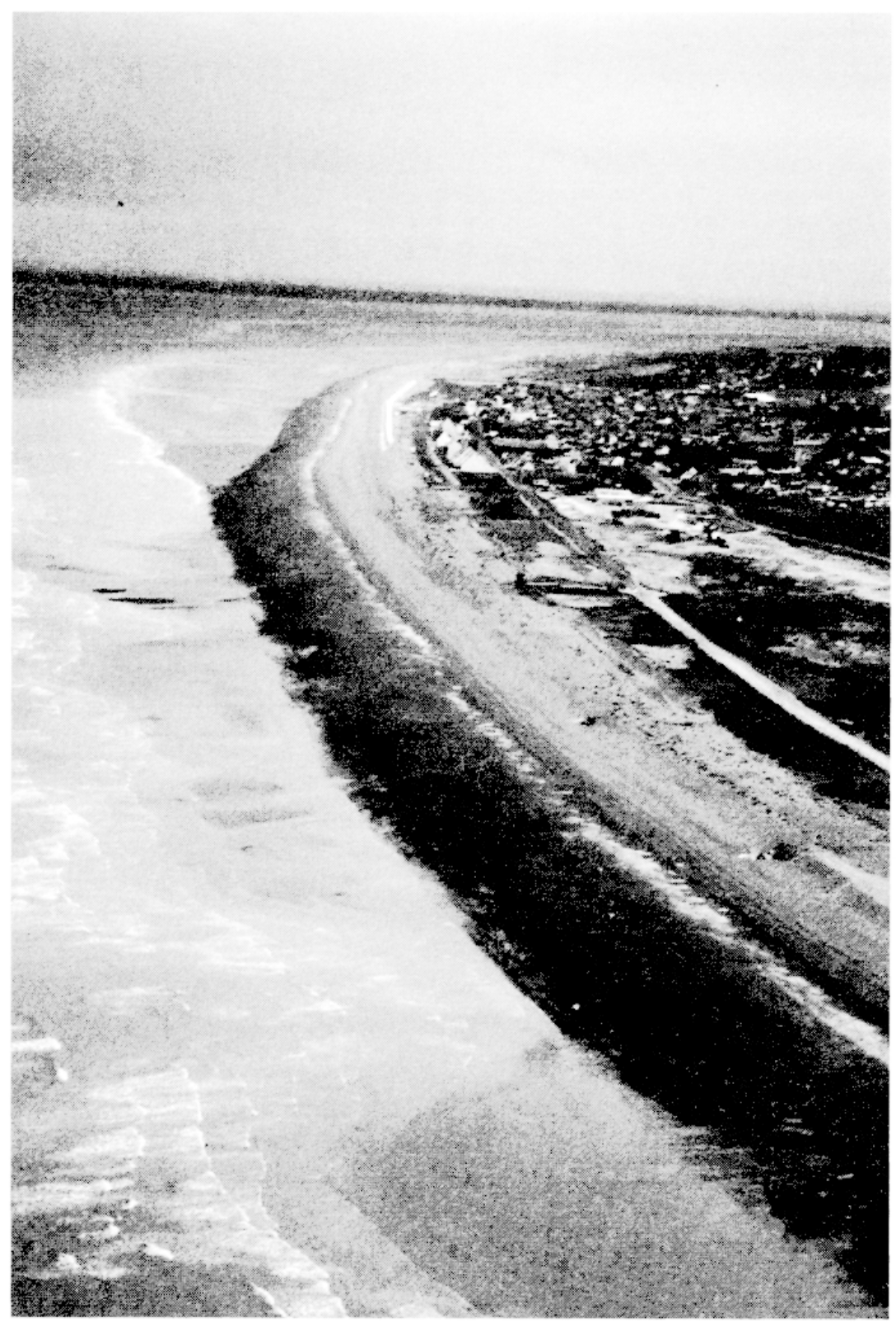

Photo 3 - L'estran sableux et le changement d'orientation du trait de côte lié à la progradation de la flèche, au nord de Cayeux-sur-Mer. Noter la réfraction accrue engendrée par l'élargissement de l'estran sableux à partir de cette station balnéaire et l'éloignement de la zone de déferlement de la houle vis-à-vis du haut estran de galets du fait de l'accrétion du bas estran sableux. En revanche, la zone de déferlement est très proche du cordon de galets au sud de Caycux là où la surface de l'estran sableux a été abaissée par l'érosion. Photo prise deux heures avant la pleine mer.

Photo 3 - The sandy foreshore and the change in shoreline orientation due to spit progradation north of the resort of Cayeux-sur-Mer. Note the enhanced wave refraction caused by the wide sandy foreshore from Cayeux northuards. The massive accretion of the sandy foreshore in this area also reduces the duration of wave action on the gravel spit at high tide while foreshore erosion further south enhances such wave action. Photo taken two hours before high water. 
INFLUENCE DE L'ESTRAN SABLEUX À MOYEN TERME

Sur une échelle de temps qui couvre le $\mathrm{xx}^{\mathrm{c}}$ siècle, il existe une nette différence de comportement morphosédimentaire entre le cordon de galets et l'estran sableux. La dynamique du premier, plage réfléchissante raide (fig. 3) est conditionnée essentiellement par la houle et la dérive littorale qu'elle induit au contact du cordon lorsque celui-ci est progressivement inondé par la marée. Sur cette plage raide, l'action de la houle est concentrée durant trois à six heures en fonction du niveau d'accrétion (au nord) ou d'abaissement (au sud) de l'estran sableux. Le but de l'article n'est pas de détailler ces processus morphodynamiques instantanés, qui ont été traités ailleurs (Dolique, 1998a) mais de considérer la manière dont l'évolution de l'estran sableux a été différente de celle du cordon, et l'influence du premier sur le second.

À l'exception du secteur proximal du cordon en érosion qui correspond aussi à un secteur d'ablation de l'estran sableux, il n'existe pas de concordance en zone distale entre les secteurs morphologiques définis pour le cordon et la tendance évolutive de la surface de l'estran sableux. L'accrétion distale sableuse recouvre un secteur en accrétion (secteur 3, fig. 2) et deux secteurs, respectivement en érosion jusqu'à une période récente (secteur 4) et en accrétion (secteur 5) par allongement de la flèche actuellement, ce dernier secteur étant relativement stable lui aussi.

Le rôle du bas de plage sableux dans l'évolution morphosédimentaire de la flèche de galets doit être considéré par rapport à trois aspects liés : (1) la fragilisation du cordon dans sa partie proximale ; $(2)$ la division de la cellule sédimentaire de la flèche en deux ; et (3) la stabilité récente des secteurs 4 et 5.

La fragilisation du cordon dans sa partie proximale tient avant tout de son bilan déficitaire en galets. Toutefois, l'abaissement de l'estran sableux dans ce secteur a pu favoriser un temps d'action de la houle sur le cordon plus long. Ceci a entraîné une exposition accrue de cette partie à la pression dynamique du déferlement et a donc exacerbé le rôle de la dérive littorale et le comportement réfléchissant très marqué de cette partie du cordon.

La division de la cellule de galets a pu être facilitée par l'accrétion généralisée qui a affecté l'estran sableux dans la zone distale du cordon (photo 3), mais ceci n'enlève en rien le rôle fondamental exercé par la houle, et les changements des conditions de la prise en charge des galets par la dérive générés par des différences de réfraction au fur et à mesure que les crochets successifs de cordons se sont mis en place dans le secteur 3 (photo 2). La division cellulaire que nous avons identifiée est liée uniquement à des gradients de transport de galets par la dérive engendrée par la houle et peut se produire dans des environnements où les flèches de galets sont dépourvues d'un estran sableux (Orford et al., 1996). L'afflux net de sables vers le nord favorise cependant la dissipation de l'énergie des vagues et donc une baisse de leur capacité à transporter des galets le long de la flèche, améliorant ainsi les conditions d'accumulation de galets dans le secteur 3. L'accumulation sableuse dans cette zone distale explique aussi la stabilisation récente des secteurs 4 et 5 où les vagues sont aujourd'hui fortement dissipées. L'afflux généralisé et massif de sables fait que ce qui reste de la plage de galets dans le secteur 4 n est atteint par les vagues qu'en période de vive-eau exceptionnelle. Dans le secteur 5. le transit continue encore, même sous de petits coefficients de marée, mais le temps de prise en charge des galets par la dérive littorale est devenu 
plus court du fait de l'accrétion importante de l'estran sableux qui met le haut estran de galets à l'abri des vagues pendant une bonne partie du cycle de marée. Cette tendance explique les faibles taux d'extension de la pointe de la flèche de galets au Hourdel (fig. 2) observés depuis dix ans (Dolique, 1998a).

\section{CONCLUSION}

Les divers travaux sur la flèche de galets des Bas-Champs de Picardie depuis un siècle ont considéré essentiellement l'évolution de ce cordon en termes de perturbations du transit de galets liées aux activités humaines. De telles perturbations ont affecté aussi le transit des sables le long du littoral, entraînant une érosion de l'estran sableux dans la partie proximale érodée de la flèche. L'effet net de l'abaissement de cet estran est une augmentation du temps de prise en charge des galets par la houle lors d'un cycle de marée. Dans les conditions de dérive littorale marquée et de pénurie de galets qui ont régné dans ce secteur, ceci a probablement exacerbé l'érosion de la flèche. Le passage de ce cordon d'une structure sédimentaire longitudinale unicellulaire à une structure bi-cellulaire est un phénomène d'adaptation directement lié aux modifications de son bilan sédimentaire à moyen terme. Comme Forbes et al. (1995) et Orford et al. (1996) l'ont récemment démontré dans leurs synthèses sur les évolutions rapides des cordons de galets des côtes canadiennes et irlandaises, de tels mécanismes peuvent impliquer des réorganisations à large échelle des stocks sédimentaires disponibles. Ces réorganisations se soldent par une déstabilisation rapide de ces corps sédimentaires, d'apparence stable et robuste avec leur composition granulométrique grossière, entraînant notamment des divisions en cellules sédimentaires plus petites.

Il est clair, à partir des exemples dans la littérature, que la division en deux ou plusieurs cellules d'une cellule sédimentaire unique ne nécessite pas la présence d'un estran sableux. Les conditions de transit et d'accumulation le long de telles flèches affectent la houle par réfraction, entraînant le dépôt des charges de galets, comme dans le secteur 3 du cordon de Cayeux, et donc l'amorce de deux cellules. Ces processus ne peuvent être que renforcés par l'importante accumulation sableuse dans ce secteur qui contribue à dissiper et à réfracter davantage la houle. Par ailleurs, l'accumulation de sables dans cette partie distale de la flèche a fini par protéger, contre la houle, le secteur en érosion de la deuxième cellule privée de galets. Il est très important de tenir compte de ces processus pour les décisions d'aménagement concernant ce type de côte, surtout lorsqu'on procède à des rechargements de galets pour lutter contre l'érosion.

\section{Remerciements}

Nous remercions Arnaud Héquette, les rapporteurs, Rémi Dalongeville et Paul Bernier, et un rapporteur anonyme pour leurs remarques qui ont aidé à améliorer le manuscrit. Denis Marin est remercié pour la réalisation des figures.

\section{Références}

Anthony E.J. (1998) - Marine sand supply from the English Channel and large-scale coastal dune formation and estuarine infill in northern France. International Comference on Coastal Enviromments : Sedimentology, Geomorphology; and Geoarchaeology; 9-10 Novembre 1998. Programme and Abstracts, The Geological Society, Londres, 12-13. 
Anthony, E.J., Cohen, (O. (1996) - Organisation et déstabilisation morphodynamique des plages de galets de la Baie des Anges. Côte d'Azur. In : Géomorphologie. risques naturels et aménagements. Mélanges en hommage à Maurice Julian. Révue d'Analyse Spatiale Quantitative et Appliquée. 38 \& 39. $29-37$.

Anthony E.J., Dubar M., Cohen (O. (1998) - Les cordons de galets de la Baie des Anges : histoire environnementale et stratigraphique : ćvolution morphodynamique récente en réponse à des aménagements. Géomorphologie : relief, processus, environnement, 1998, n² 2,167-188.

Beauchamp J. (1994) - Abrasion des galets et origine du sable en baie de Somme. Travaux du Dípartement de Géologic de l'Université de Picardie. 3. 43-59.

Beauchesne, P., Courtois, G. (1967) - Etude du mouvement des galets le long de la cote des BasChamps de la Somme. utilisation de traceurs radioactifs. Cahiers Océanographiques. 19. 61.3-625.

Bray M.J., Hooke J.M., Carter D.J. (1995) - Littoral cell definition and budgets for central southern England. Journal of Coastal Research. 11. 381-400).

Briquet A. (1930) - Le littoral du Nord de la France et son évolution morphologique. Armand Colin, Paris. France. 439 p.

Carter R.W.G., Orford J.D. (1993) - The morphodynamics of coarse clastic beaches and barriers : a short - and long-term perspective. Journal of Coastal Research. Special Issue, 15, 158-179.

Costa S. (1997) - Dynamique sédimentaire et risque naturel : l'impact des variations du niveau marin. des aménagements et des modifications climatiques entre la Baie de Seine et la Baie de Somme. Thèse. Université de Paris I. $376 \mathrm{p}$.

Dallery F. (1955) - Less rivages de la Somme. autrefois, aujourd'hui et demain. Mémoires de la Société d'Emulation Historique et Littéraire d'Abbeville. A. et J. Picard et Cie. Paris. 307 p.

De Batist M., Marsset T., Missian T., Reynaud J., Tessier B., Chamley H. (1993) - Large - and small-scale internal structures of the Bassure de Baas Bank : recording by high resolution seismics and sub-bottom profiling. Rapport Annuel Projet MAST-Starfish. 77-83.

Dolique F. (1998a) - Dynamique morphosédimemtaire ét aménagements induits du littoral picard au Sud de la Baie de Somme. Thèse, Université du Littoral "Côte d'Opale", Dunkerque. France, $417 \mathrm{p}$.

Dolique, F. (1998b) - Conflits d'usage et gestion d'un littoral anthropisé : les Bas-Champs de Cayeux (Somme). Larus, 7. 23-26.

Dolique F. (1998c) - Images des changements d'un littoral : les Bas-champs de Cayeux (Somme). Mappemonde. 50, 1998/2, 36-39.

Dolique F., Anthony, E.J. (1998) - The gravel barrier of Cayeux-sur-Mer, Picardy, France : a summary of recent morphosedimentary changes. Journal de Recherche Océanographique, 23, 157-162.

Dupont J.-P. (1994) - Le site du Cap Hornu. arrêt $\mathrm{n}^{\circ}$ 4. Travaux du Département de Géologie de l'Université de Picurdie, 3, 25-27.

Forbes D.I.., Orford J.D., Carter R.W.G., Shaw J., Jennings S.C. (1995) - Morphodynamic evolution. self-organisation. and instability of coarse-clastic barriers on paraglacial coasts. Marine Geology. 126. 6.3-8.5.

Grochowski N.T.L., Collins M.B., Boxall S.R., Salomon J.C., Breton M., Lafite R. (1993a) Sediment transport pathways in the eastern English Channel. Oceanologica Acta, 16. 531-537.

(Grochowski N.T.L., Collins M.B., Boxall S.R., Salomon J.C. (1993b) - Sediment transport predictions for the English Channel. using numerical models. Journal of the Geological Society, 150, 683-695.

Hachache, N. (1998) - Laménagement des baies : une priorité : l'accès maritime. Le Moniteur des traraux publices "t du bâtimentı, 4942, 14 août 1998. 10-11.

Hascoët M. (1988) - Evolution du littoral picard. entre le village d’Ault et le port du Hourdel (Somme), élude photogéomorphologique. Colloque Mer et Littoral. Couple à Risque. Ministère de L'Environnement. Paris. 465-477.

Levoy F., Anthony E..J., Barusseau J.-P., Howa H., Tessier B. (1998) - Morphodynamique d une plage macrotidale à harres. Compte Rendus de l'Académie des Sciences de Paris, Sciences de la terre et des planieses, 327, $811-818$.

May J.P., Tanner W.F. (1973) - The littoral power gradient and shoreline changes. In : Coastal Geomorpholog! D.R. Coates. (ed). Lniversity of New York. Binghampton. L.S.A.. 43-60.

Migniot C., Bellesort B. (1974) - Bas-Champs de la Somme, protection contre la mer. étude sédimentologique complémentaire, étude sur plan des solutions de stabilisation. Rapport LCHF pour la Direction Départementale de l'Environnement de la Somme. Abbeville. France. 55 p. 
Orford J.D., Carter R.W.G. (1995) - Identification of mesoscale forcing of a coastal gravel-dominated barricr. Marine Geology, 126. 201-212.

Orford J.D., Carter R.W.G., Jennings S.C. (1996) - Control domains and morphological phases in gravel-dominated coastal barriers of Nova Scotia. Journal of Coastal Research, 12, 589-60)4.

Queffeullou G. (1992) - Le littoral des Bas-Champs : un cas de risque majeur littoral. Mémoire de D.E.S.S., Génie Géologique, Université d'Orsay, Paris, France, 125 p.

Regrain R. (1970) - Le littoral des Bas-Champs, au Sud de la Somme. CRDP, Amiens, 27 p.

Regrain R. (1971) - Etude géographique, essai de géomorphologie statiqué, cinćmatique et dynamique du littoral picard. CRDP. Amiens. $107 \mathrm{p}$.

Regrain R. (1992) - Protéger le littoral ouest des Bas-Champs de Cayeux?' In : Les littoraux en France : risques et aménagements. CRDP Picardie, 49-73.

Regrain, R., Vignon F., Wattez J.R. (1979) - Un secteur côtier d'intérêt scientifique particulier : les Bas-Champs de Cayeux (Somme). Publications Scientifiques et Techniques du CNEXO, Colloque ${ }^{\circ} 2$, Brest, 165-176.

SOGREAH (1995a) - Littoral des Bas-Champs: arant-projet sommaire de la solution retenue. Rapport, Direction Départementale de l'Equipement de la Somme. Abbeville, France. 65 p.

SOGREAH (1995b) - Etude sédimentologique de la Baie de Somme. Sinthèse des données naturelles. Rapport, Direction Départementale de l'Equipement de la Somme. Abbeville. France, 65 p.

SOGREAH (1997) - Conclusions de l'expérimentation sur modèle réduit de l'ensablement de la Baie de Somme. Prévisions à moyen ét long termé et propositions d'aménagement. Rapport. Direction Départementale de l'Environnement de la Somme. Abbeville. France. $129 \mathrm{p}$.

Tessier B. (1997) - Expressions sédimentaires de la dynamique tidale. Mémoire d'Habilitation à Diriger des Recherches. Sciences Naturelles, Université des Sciences et Technologies de Lille.

Manuscrit reçu le 16 Octobre 1998. accepté le 18 Février 1999 